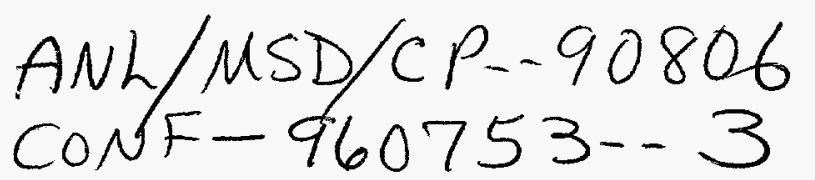

SFP 191996

OSTI

\title{
RELATIONSHIP BETWEEN NANOCRYSTALLINE AND AMORPHOUS MICROSTRUCTURES BY MOLECULAR DYNAMICS SIMULATION*
}

\author{
P. Keblinski, S. R. Phillpot and D. Wolf \\ Materials Science Division \\ Argonne National Laboratory \\ Argonne, IL, 60439, USA \\ H. Gleiter \\ Forschungszentrum Karlsruhe \\ 76021 Karlsruhe, Germany
}

\section{AUGUST 1996}

\begin{abstract}
The submitted manuscript has been created by the University of Chicago as Operator of Argonne National Laboratory ("Argonne") under Contract No. W-31-109-ENG-38 with the U.S. Department of Energy. The U.S. Government retains for itseif, and others acting on its behalf, a paid-up, nonexclusive, irrevocable worldwide license in said article to reproduce, prepare derivative works, distribute copies to the public, and perform publicly and display publicly, by or on behall of the Government.
\end{abstract}

Manuscript of an invited lecture presented at the Third International Conference on Nanostructured Materials, Kona, Hawaii, July 8-12, 1996 and to be published in Nanostructured Materials.

${ }^{*}$ The work was supported by US Department of Energy BES-Materials Science under Contract No. W-31-109-Eng-38. 


\section{DISCLAIMER}

Portions of this document may be illegible in electronic image products. Images are produced from the best available original document. 


\section{DISCLAIMER}

This report was prepared as an account of work sponsored by an agency of the United States Government. Neither the United States Government nor any agency thereof, nor any of their employees, makes any warranty, express or implied, or assumes any legal liability or responsibility for the accuracy, completeness, or usefulness of any information, apparatus, product, or process disclosed, or represents that its use would not infringe privately owned rights. Reference herein to any specific commercial product, process, or service by trade name, trademark, manufacturer, or otherwise does not necessarily constitute or imply its endorsement, recommendation, or favoring by the United States Government or any agency thereof. The views and opinions of authors expressed herein do not necessarily state or reflect those of the United States Government or any agency thereof. 


\title{
RELATIONSHIP BETWEEN NANOCRYSTALLINE AND AMORPHOUS MICROSTRUCTURES BY MOLECULAR DYNAMICS SIMULATION
}

\author{
P. Keblinski a, S. R. Phillpot ${ }^{a}$, D. Wolf a and H. Gleiter b \\ a Materials Science Division, Argonne National Laboratory, Argonne, IL 60439, USA.

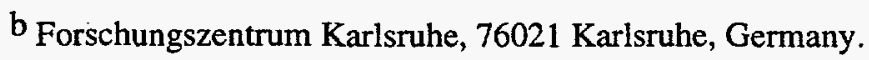

\begin{abstract}
A recently developed molecular-dynamics simulation method for the growth of fully dense nanocrystalline materials by crystallization from the melt was used together with the Stillinger-Weber three-body potential to synthesize nanocrystalline silicon with a grain size up to $75 \AA$. The structures of the highly-constrained grain boundaries (GBs), triple lines and point grain junctions were found to be highly disordered and similar to the structure of amorphous silicon. These and our earlier results for fcc metals suggest that a nanocrystalline microstructure may be viewed as a two-phase system, namely an ordered crystalline phase in the grain interiors connected by an amorphous, intergranular, glue-like phase. The analysis of the structures of bicrystalline GBs in the same materials reveals the presence of an amorphous intergranular equilibrium phase only in the high-energy but not the low-energy GBs, suggesting that only high-energy boundaries are present in nanocrystalline microstructures.
\end{abstract}

\section{INTRODUCTION}

The atomic structures of the grain boundaries (GBs) in nanocrystalline materials and their relationship to amorphous microstructures have been the subject of intense debate ever since the first ultrafine-grained polycrystals were synthesized over a decade ago by consolidation of small clusters formed via gas condensation. However, in spite of much experimental work on the structure and properties of nanocrystalline materials performed to date, neither a structural model for the GBs nor a thermodynamics-based description consistent with the observations has evolved. Of particular interest has been the relationship of the structures of the GBs in nanocrystalline materials to amorphous structures and the possibility of a (sharp or continuous) transition between them. For example, Shen et al. [1] demonstrated that ball milling can be used to produce two-phase nanocrystalline and amorphous silicon; for an average grain size of 8 $\mathrm{nm}$, about $15 \%$ of the material was identified as being amorphous [1]. Extensive earlier work by Veprek et al. [2] on nanocrystalline silicon had also identified a strongly grain-size dependent amorphous component. An inherent limitation of these studies is the difficulty in determining the spatial distribution of the amorphous component within these highly inhomogeneous microstructures; i.e., to answer the question as to whether entire grains are amorphous or whether perhaps only the GBs and grain junctions are amorphous. 
To develop a structural model and also to elucidate the connection between amorphous and nanocrystalline microstructures, we have recently developed a molecular-dynamics (MD) simulation method for the growth of fully dense nanocrystalline materials from the melt into which small crystalline seeds with more or less random orientations were inserted. [3] In spite of some similarities to the well-known structures of the interfaces in coarse-grained materials, the atomic structures of the GBs thus obtained exhibited a virtually complete lack of long-range structural periodicity parallel to the interfaces because many short segments with locally different atomic structures were observed to coexist in these highly constrained microstructures. [4] Based on these observations, we suggested that historic models viewing the grain boundaries in nanocrystalline materials as an "amorphous-cement"-like phase [5] with a more or less uniform width, density and energy density describe the interfaces more aptly than structure models derived from atomic-resolution experiments on coarse-grained materials. Also, from simulations of the phonon density of states and of the related free energy $[6,7]$ we were able to demonstrate that below a certain critical grain size (of typically $1.5-2 \mathrm{~nm}$ ) nanocrystalline microstructures should be thermodynamically unstable with respect to the amorphous phase.

In this paper we further investigate the atomic structures of the GBs in nanocrystalline materials and their relationship to the amorphous phase. By contrast with our earlier simulations $[3,4,6,7]$ involving a generic fcc-metal (Lennard-Jones) interatomic potential, here we focus on nanocrystalline silicon. For several reasons silicon represents an ideal choice of a one-component covalent model material for this simulation study. First, a well-tested interatomic potential due to Stillinger and Weber [8] (SW) is available which provides not only a good description of the diamond and amorphous phases but also of the six-coordinated structure of the liquid [9] and the melting temperature (of $1690 \mathrm{~K} \pm 20 \mathrm{~K}$, remarkably similar to the experimental value $1683 \mathrm{~K}$ ). Second, the structure and excess energy of bulk amorphous $\mathrm{Si}$ are well reproduced by the SW potential [9-11]. Third, the SW potential has been used successfully to describe crystalline silicon in a wide variety of defective environments ranging from small clusters [12] and GBs [13] to the structure and dynamics of dislocations [14]. Finally, the SW potential successfully describes the faceting of the (100) crystal-liquid interface [15] and hence permits simulation of homo-epitaxial crystal growth from the melt [16]. We believe this combination of relevant phase, defect and kinetic information reproduced at least semiquantitatively by the SW potential makes Si an ideal model material for our purpose.

\section{NANOCRYSTALLINE SILICON}

The three-dimensionally (3d) periodic cubic simulation cell used in our simulations is sketched in Fig. 1. The starting configuration consists of an fcc arrangement of four randomly oriented seed grains of diamond structured Si embedded in the melt filling the rest of the cell. Due to the significant number of atoms in the initial seeds (see below), the Brownian motion of the seeds in the melt is negligible on an MD time scale [3] although, in principle, the seeds are free to move as a whole. The resulting microstructure consists of four, approximately dodecahedral grains delimited by 24 distinct GBs, 32 triple lines, 8 four-fold and 4 six-fold point junctions [17]. The random grain misorientations usually result in GBs having both tilt and twist components; i.e., "general" boundaries (by contrast with symmetric or with "special" GBs). The fcc arrangement of the seeds represents an improvement over our pervious work [3] 
in which the seeds were placed on a simple cubic lattice, resulting in a cubic grain shape and rather unrealistic four-fold line junctions.

The crystal-growth simulation starts by melting the system at $3000 \mathrm{~K}$ and constant volume for 1000 integration time steps $\left(\Delta t=2.75 \times 10^{-15} \mathrm{~s}\right)$ while the seed atoms are kept fixed at their perfect-crystal positions. In the next $1000 \mathrm{MD}$ steps the four seeds are gradually rotated to randomly chosen orientations. After further equilibration at high temperature the system is cooled down to $T=1250 \mathrm{~K}$, (i.e., well below the melting point) in order to generate a thermodynamic driving force, $\mathrm{T}_{\mathrm{m}}-\mathrm{T}$, for crystal growth. A constant-pressure algorithm is applied to relax the system to zero external pressure and the system is allowed to evolve freely with no constraints imposed on the seed atoms. The growth continues until the internal energy of the system stops decreasing; depending on the system size, this growth phase requires typically $150,000-300,000 \mathrm{MD}$ time steps (i.e., up to $10^{-9} \mathrm{~s}$ of real time). Finally the system is "slowly" cooled down to $\mathrm{T}=0 \mathrm{~K}$ (over $\sim 20,000$ time steps) under zero external pressure.

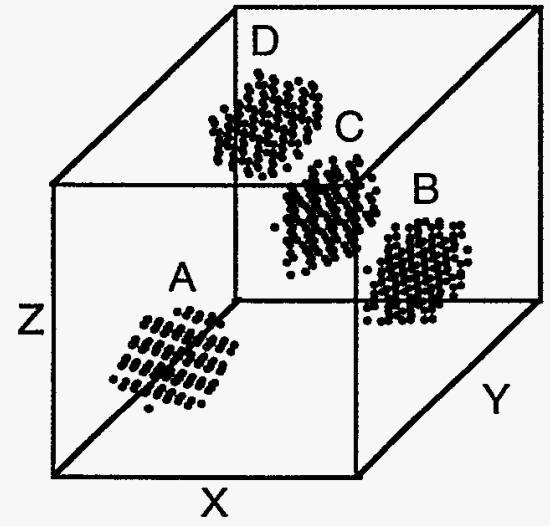

Fig. 1. Cubic, 3d periodic simulation cell containing four randomly oriented seed grains arranged on an fcc lattice and embedded in the melt.

Nanocrystalline microstructures with three different grain diameters were synthesized by this method: $\mathrm{d}=3.8 \mathrm{~nm}, 5.4 \mathrm{~nm}$ and $7.3 \mathrm{~nm}$ corresponding, respectively, to 8000,21952 and 54872 atoms in the simulation cell. The size of the initial seeds was scaled with the system size such that $15 \%$ of the atoms constituted the seeds while the remaining $85 \%$ represented the melt.

To characterize the fcc microstructures thus obtained, we investigate planar cuts of thickness $0.5 a_{0}$ through the simulation cell $\left(a_{0}=5.43 \AA\right.$ is the zero-temperature lattice parameter). Figure 2 
shows gray-scale contours of equal energy per atom for a slice parallel to the micro-structural (111) planes for the system with the intermediate grain size; this cut slices through all four grain centers (see also Fig. 1). Clearly, all GBs (seen as dark lines) have roughly the same width while the triple lines (seen as points) appear to be slightly wider than the GBs. These structural features remain the same when changing the grain size; in particular, the spatial extent of the disorder at the GBs and grain junctions is practically independent of the grain size. [17]

The fact that the disorder at the triple lines and point grain junctions is spatially more extended than at the GBs is not too surprising because, during the growth process, GBs are created first. The eventual formation of triple lines and, finally, point junctions occurs under the ever increasing constraints of an already existing solid matrix, giving the atoms in these regions less and less freedom to homo-epitaxially attach themselves to already crystallized material and to relax the local excess volume stored in these highly disordered regions.

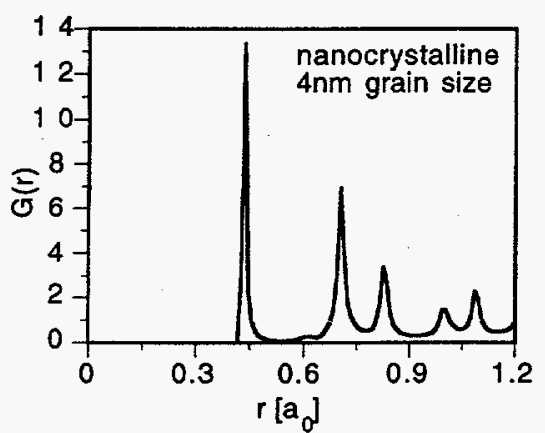

Fig. 3. System-averaged radial distribution function, $G(r)$, for the model nanocrystalline material with the smallest grain size $(\sim 3.8$ $\mathrm{nm}) . \mathrm{G}(\mathrm{r})$ is normalized such that for large $r$ the average atomic density is one.

Figure 3 shows the system-averaged radial distribution function, $G(r)$, for the system with the smallest grain size $(\sim 3.8 \mathrm{~nm})$. According to Fig. 3, even for the smallest grain size $G(r)$ exhibits sharp crystalline peaks originating from the ordered grain interiors. However, between the crystalline peaks a non-vanishing background is clearly visible which, as we shall show, is due to the disordered regions along the GBs and grain junctions.

The local atomic structure in the inhomogeneous regions of the material may be characterized by local radial distribution functions, such as those shown in Fig. 4 associated with the GBs, triple junctions, and the four- and six-fold point-junctions. (The variations in these functions from one GB or grain junction to another are within the scatter of the data.) Most notably, all these local distributions are remarkably similar to the overall $\mathrm{G}(\mathrm{r})$ of bulk amorphous silicon also shown in Fig. 4, indicating a virtually complete absence of long-range order in these locally disordered environments. In particular, the second crystalline peak is broadened while the third crystalline peak has disappeared altogether, by contrast with the system-averaged $G(r)$ in Fig. 3 in which these peaks are clearly present.

The GB energy varies relatively little from one boundary to another, although for any given boundary there is some spatia variation within the GB plane [3]. Moreover, the average GB energy was found to be essentially independent of the grain size and equal to 1300-1350 $\mathrm{erg} / \mathrm{cm}^{2}$, with none of the $24 \mathrm{GBs}$ having an energy lower than about $900 \mathrm{erg} / \mathrm{cm}^{2}$ or higher than about $1500 \mathrm{erg} / \mathrm{cm}^{2}$. This range of values is considerably narrower than the GB energies determined, by lattice relaxation at zero temperature, for twist boundaries on the three densest planes of Si. [13] The absence of low-energy GBs probably has two causes. First, the random misorientations between grains effectively avoid the formation of low-angle and of "special" GBs since all GBs in the system have both tilt and twist components and hence a relatively high 


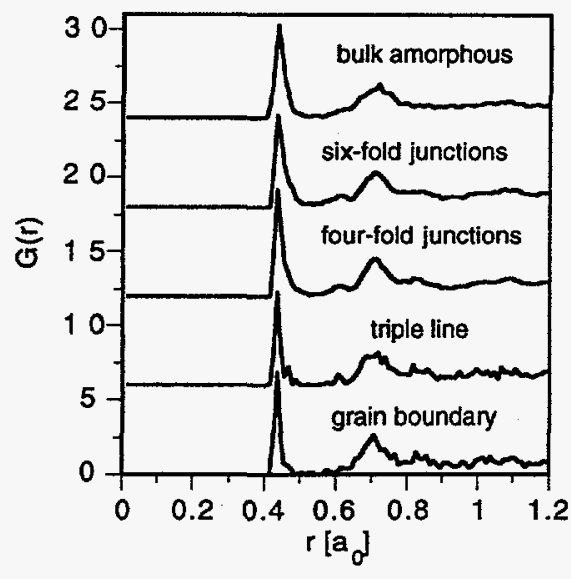

Fig. 4. Local radial distribution functions in the GBs and grain junctions for the microstructure with the intermediate grain size $(\sim 5.4 \mathrm{~nm})$. For comparison, $\mathrm{G}(\mathrm{r})$ for bulk amorphous silicon is also shown.

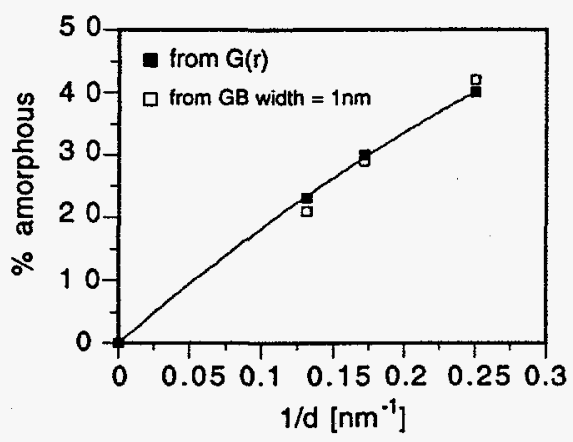

Fig. 5. Percentage of the amorphous phase as a function of the inverse grain size (1/d) obtained from the analysis of the radial distribution function, $G(r)$, and via the width (of $\sim 1 \mathrm{~nm}$ ) of the amorphous GB region and the total GB area in the system. The solid line represents a quadratic least-squares fit through the origin for the $G(r)$ data. energy. [18] Second, whereas in a bicrystal rigid-body translations parallel to the GB are known to provide a powerful relaxation mechanism, in severely constrained microstructures these translations cannot be optimized simulta-neously for all the grains and, hence, all the GBs; this results in higher GB energies [3].

The fraction of the disordered material in the GBs and grain junctions can now be estimated given that all GBs exhibit practically the same (amorphous) structure and that the GB width is practically independent of the grain size. Assuming, for example, a GB width of 1 $\mathrm{nm}$, the three grain sizes give values of 0.42 , 0.29 and 0.21 , respectively, for the volume fraction of amorphous material. [17] Alternatively, this fraction can be estimated from the non-vanishing background between the crystalline peaks in the overall $\mathrm{G}(\mathrm{r})$ in Fig. 4 , giving the volume percentage of the amorphous material, plotted in Fig. 5 against the inverse grain size, $1 / \mathrm{d}$. (The latter is a more natural length scale than $d$ as it corresponds roughly to the ratio of the total GB area to the system volume.) According to Fig. 5 , the two methods used to extract the fraction of amorphous material give similar results. The curvature in the least-squares fit to the $G(r)$ data is due to an increasingly important contribution from the grain junctions as the grain size decreases.

The above analysis suggests a universal local atomic structure and excess energy of the GBs and grain junctions that is virtually indistinguishable from that of bulk amorphous silicon. The above simulations, in addition, provide information on the spatial distribution of the amorphous material within the microstructure. Moreover, these simulations suggest that under the constraints of the nanocrystalline microstructure such an amorphous intergranular "phase" is present in thermodynamic equilibrium which cannot be eliminated during high-temperature anneal, unless the temperature is high enough to initiate grain growth that ultimately leads to lower-energy GBs. The detailed atomic structures of the 
GBs and grain junctions may therefore not be of great importance in such highly constrained microstructures. In fact, a two-phase model in which the inverse grain size (i.e., ratio of the GB area to the system volume) is a natural variable describes our results rather well. That in nanocrystalline $\mathrm{Si}$ a strongly grain-size dependent fraction of the material is, indeed, amorphous is consistent with experiments $[1,2]$.

The above results confirm the conclusions drawn from our previous simulations of a nanocrystalline model fcc metal described by the Lennard-Jones potential, in spite of the cubic grain shape and the simple-cubic grain arrangement in these first simulations [3]. Furthermore, recent simulations of nanocrystalline $\mathrm{Pd}$ [19] involving an embedded-atom-method potential and the same fcc microstructure and dodecahedral grain shapes as in the above Si simulations yielded a two-phase microstructure virtually identical to the one obtained here for $\mathbf{S i}$, including disordered GBs of uniform width and crystalline grain interiors. However, by contrast with $\mathrm{Si}, \mathrm{x}$ ray experiments on nanophase $\mathrm{Pd}$, albeit with a much larger grain size than that considered in the simulations, give no indication for the presence of a disordered intergranular phase. [20]

\section{AMORPHOUS INTERGRANULAR FILMS IN SILICON BICRYSTALS}

To elucidate the origin of a thermodynamically stable, amorphous intergranular phase present in our model nanocrystalline microstructures, we have investigated the atomic structures of bicrystalline Si GBs following high-temperature equilibration. [21] Because (i) the GBs in our model nanocrystalline materials have relatively high energies and (ii) low-energy ("special") Si GBs are well known to be crystallographically ordered [22], our focus is on the role of the GB energy on the structure of bicrystalline GBs. As candidate high-energy boundaries we have studied high-angle symmetric twist boundaries which are known to have generally higher energies than symmetric tilt boundaries [13]; they therefore appear more likely to disorder while lowering their energy upon high-temperature equilibration than do tilt boundaries. Their fully relaxed zero-temperature MD input structures were obtained by static iterative energy minimization.

To equilibrate the GB structure at elevated temperature, the bicrystal was subjected to two distinct high-temperature treatments. In the first treatment the GB region was melted at $3000 \mathrm{~K}$; the temperature is then lowered below the melting point to $1350 \mathrm{~K}$ to initiate re-crystallization from the planar seeds provided by the unmelted perfect-crystal material in the simulation cell. This re-crystallization required typically about $15,000 \Delta t$ after which the system was kept at that temperature for an additional 10,000 $\Delta \mathrm{t}$. The final structure was then obtained by cooling the system down to zero temperature at a rate of $1 \mathrm{~K}$ per $50 \Delta \mathrm{t}$, i.e. at a cooling rate of $7.25 \times 10^{12}$ $\mathrm{K} / \mathrm{s}$ (which is rather "slow" on an MD time scale but many orders of magnitude higher than experimental cooling rates). In the second treatment the GB was simply annealed at $\mathrm{T}=1500 \mathrm{~K}$ $\left(190 \mathrm{~K}\right.$ below $\mathrm{T}_{\mathrm{m}}$ ) for $20,000 \Delta t$ and then cooled down to zero temperature. In all cases the two treatments yielded GB structures with practically the same overall characteristics (radial and bond-angle distribution functions and energy profiles), although the detailed atomic structures and GB energies were not completely identical. To verify that structures thus obtained were, indeed, fully equilibrated they were re-heated up to $1500 \mathrm{~K}$, kept at this temperature for an additional 20,000 $\Delta t$ and then again cooled down, revealing no changes in these characteristics. 


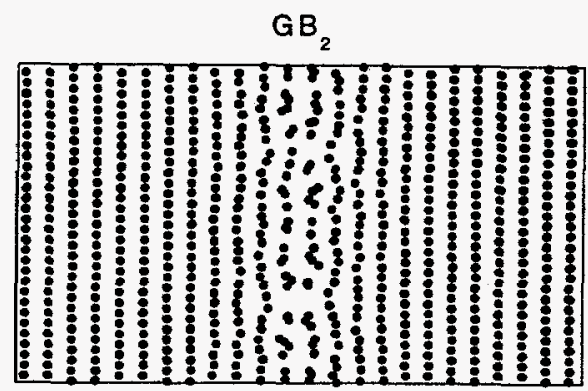

$G B$
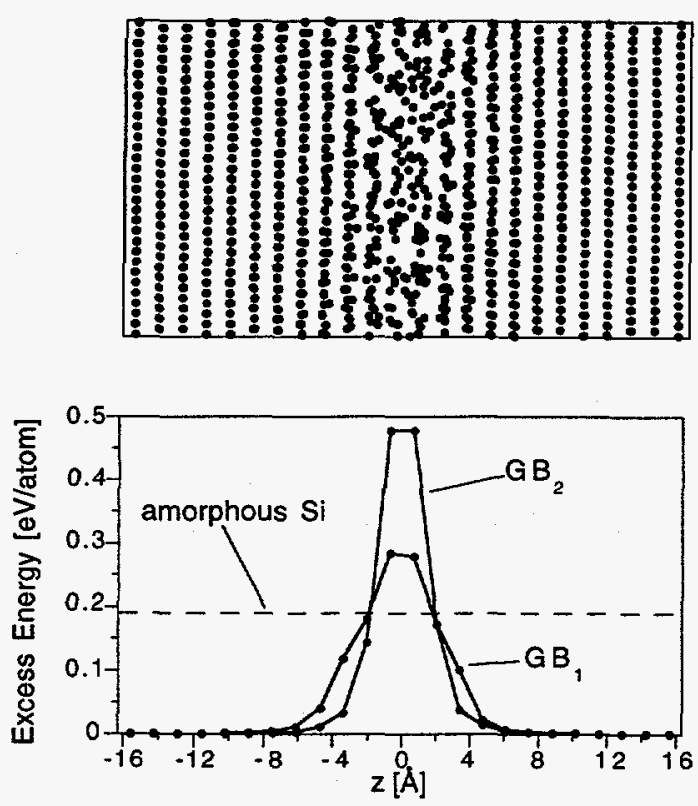

Fig. 6. Projected positions of the atoms for the (100) $\phi=43.60^{\circ}(\Sigma 29)$ twist GB in Si. Upper panel: zero-temperature relaxed structure $\left(\mathrm{GB}_{2}\right)$; center: high-temperature relaxed structure $\left(\mathrm{GB}_{1}\right)$. Lower panel: average energy per atom in (100) slices of thickness $0.25 \mathrm{a}_{0}$ as a function of distance, $z$, from the GB. The dashed line shows the cohesive energy of bulk amorphous Si for the SW potential.
We first present in some detail results for the $(100) \phi=43.60^{\circ}(\Sigma 29)$ twist GB. Its zero-temperature relaxed $\left(\mathrm{GB}_{2}\right)$ and high-temperature equilibrated $\left(\mathrm{GB}_{1}\right)$ structures are shown in the top and center panels in Fig. 6. The $\mathrm{GB}_{2}$ structure has a relatively high energy of $1464 \mathrm{erg} / \mathrm{cm}^{2}$. [13] (Although this value represents the lowest-energy rigid-body translation of this particular GB, we found that it is rather insensitive to such translations.) The related plane-by-plane average-energyper-atom profile is shown in the bottom panel. While the $\mathrm{GB}_{2}$ profile is rather narrow with a maximum value of $-\mathbf{3 . 8 7}$ $\mathrm{eV}$, the $\mathrm{GB}_{1}$ profile is broader but its peak value is only $-4.05 \mathrm{eV}$. Despite the $\mathrm{GB}_{1}$ energy profile being broader, the related GB energy of $1340 \mathrm{erg} / \mathrm{cm}^{2}$, is approximately $10 \%$ lower than the energy of $\mathrm{GB}_{2}\left(1464 \mathrm{erg} / \mathrm{cm}^{2}\right)$. This demonstrates that high-temperature equilibration lowers the GB energy, suggesting a thermodynamic origin for this restructuring. (For later reference, the cohesive energy of bulk amorphous silicon prepared by slow cooling of the liquid [9] is $-4.15 \mathrm{eV}$; as indicated by the dashed line in the lower panel, this value is $0.185 \mathrm{eV}$ above the perfect-crystal cohesive energy of $-4.335 \mathrm{eV}$ ).

The projected structures in Fig. 6 demonstrate that the lowering of the energy is accompanied by structural disordering. Whereas $\mathrm{GB}_{2}$ exhibits a clearly identifiable planar structure even right at the GB, individual atomic planes cannot be clearly defined in the center of $\mathrm{GB}_{1}$.

The loss of crystalline order in the center of $\mathrm{GB}_{1}$ is also evident from the related local radial and bond-angle distribution functions [21] which are strikingly similar to those of bulk amorphous Si (see also Fig. 4). Furthermore, consistent with results for bulk amorphous Si using the same potential [11], in the disordered region of $\mathrm{GB}_{1}$ only about $1.5 \%$ of the atoms are 
3-fold coordinated (i.e., atoms with dangling bonds) while $83.5 \%$ are 4 -fold and $15 \%$ are 5 -fold coordinated. By comparison, the $\mathrm{GB}_{2}$ structure is considerably less-well coordinated, with $6 \%$ of the atoms having dangling bonds. [21]

For the following three reasons we believe that the amorphous film at this particular GB, indeed, represents its lowest-energy structure. First, two fundamentally different hightemperature treatments (growth from the melt and high-temperature anneal) yield practically the same amorphous GB structures. Second, subjected to further annealing these structures were found to be stable on an MD time scale. Third, these amorphous GB films are far better coordinated than the corresponding zero-temperature relaxed GBs.

The origin of the driving force for the GB disordering process is apparent from the bottom panel of Fig. 6. The peak energy of $-3.87 \mathrm{eV} /$ atom for the $\mathrm{GB}_{2}$ structure far exceeds the average energy per atom in bulk amorphous $\mathrm{Si}$ (of $-4.15 \mathrm{eV}$; dashed line). By contrast, in the most disordered plane of $\mathrm{GB}_{1}$ the average energy is only $-4.05 \mathrm{eV} / \mathrm{atom}$, much closer to the cohesive energy of amorphous Si. More importantly, the GB energy (i.e., the integral under the peak) is lowered during disordering - despite a broadening of the GB. These observations allow us to identify a criterion for the existence of an equilibrium disordered GB film: if atoms in an ordered GB have significantly higher energies than the atoms in the bulk amorphous phase, the introduction of an amorphous film into the crystalline interface is energetically favorable. By contrast, as demonstrated in Ref. 21 , if the atoms in an ordered GB have energies similar to, or lower than, the energies of the atoms in the bulk amorphous phase, the GB will not disorder.

Although the structure of the intergranular disordered film is very similar to that of bulk amorphous silicon (see Fig. 4), the film is nevertheless highly confined between the two crystalline grains. Due to this confinement, the energy of the atoms in the most disordered plane of $\mathrm{GB}_{1}$ does not decrease all the way down to the bulk amorphous value (bottom panel in Fig. 6). Moreover, detailed analysis of the related plane-by-plane structure factor [21] reveals the presence of a small - but reproducible - signal of residual crystallinity in the most disordered planes of $\mathrm{GB}_{1}$ due to the adjacent crystalline material.

Based on these insights, one might expect that all high-energy GBs should have similar, "confined-amorphous" structures. To test this prediction we investigated three other high-angle, high-energy twist boundaries with qualitatively different zero-temperature relaxed structures: $(100) \phi=61.93^{\circ}(\Sigma 17),(110) \phi=44.00^{\circ}(\Sigma 57)$ and (112) $\phi=35.26^{\circ}(\Sigma 35)$. We found that high-temperature relaxation lowers the energies of all these GBs, to values in the range of 1300 $1370 \mathrm{erg} / \mathrm{cm}^{2}$. Moreover, despite being sandwiched between different crystallographic surfaces, all GBs were found to be disordered and very similar to each other, as is also apparent from the related energy profiles in Fig. 7 (which are remarkable similar to those obtained for the GBs in nanocrystalline Si [17]). Furthermore, as for the (100) $\Sigma 29 \mathrm{~GB}$ the local radial and bond-angle distribution functions for all GBs are very similar to those of bulk amorphous Si [21].

By contrast with these disordered structures for high-energy GBs, one might expect that low-energy GBs should not lower their energy upon high-temperature equilibration and hence should not disorder. To confirm this prediction we studied the (111), $\phi=42.10^{\circ}(\Sigma 31)$ twist GB, also a high-angle GB, however on the most widely-spaced (and hence, lowest-energy [13]) plane in the diamond structure, with a zero-temperature relaxed energy of only $638 \mathrm{erg} / \mathrm{cm}^{2}[10]$. Indeed, as expected, neither its structure nor its energy change during high-temperature annealing and the GB remains crystalline right up to the GB plane. 


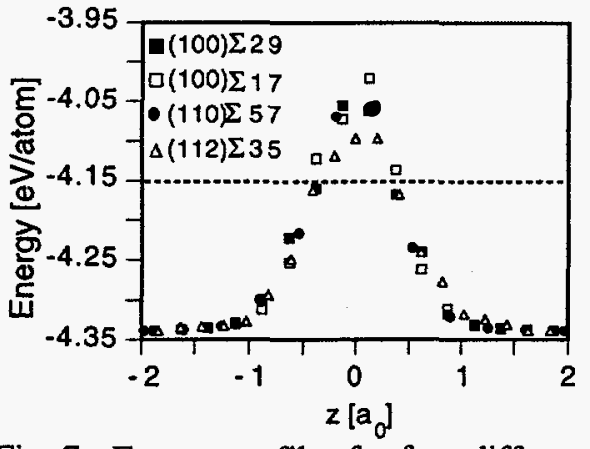

Fig. 7. Energy profiles for four different highenergy, high-angle twist GBs (see also Fig. 6). These profiles are remarkable similar to those obtained for the GBs in nanocrystalline $\mathrm{Si}$. [17]
Qualitatively similar results were obtained in our studies of symmetrical twist GBs in Pd [19]. Low-energy twist GBs on the (111) and (100) planes (the two densest planes in the fcc structure) exhibit ordered structures even after high temperature treatment, whereas high-energy, high-angle GBs on the (110) plane show disordered structures even at zero temperature, with high-temperature equilibration giving rise to further disordering accompanied by a lowering of the GB energy. [19]

\section{CONCLUSIONS}

The idealized, fully-dense, melt-grown nanocrystalline $\mathrm{Si}$ and $\mathrm{Pd}$ microstructures synthesized and characterized above not only involve more realistic (i.e., material specific) interatomic potentials than our first simulations $[3,4]$ but also exhibit a dodecahedral grain shape and an fcc arrangement of the grains, i.e., they represent a more realistic description of the structures of the line and point grain junctions. These technical improvements do not, however, change the main result of our earlier work, namely that the microstructure consists of ordered grain interiors joined by an amorphous, glue-like intergranular phase that "wets" the grain boundaries and grain junctions. As from our earlier work we therefore conclude that, given more or less random grain orientations and the absence of a distribution in the grain size, Rosenhain's historic model [5] viewing the grain boundaries as an "amorphous-cement"-like phase with a more or less uniform width, density and energy density describes the interfaces in these nanocrystalline microstructures very well.

From the comparison of the GBs in nanocrystalline Si with bicrystalline Si GBs grown from the melt or annealed at high temperatures, two conclusions can be drawn.

First, the appearance of amorphous intergranular films in high-energy boundaries with a uniform thickness and energy (i.e., independent of the grain misorientations) represents a thermodynamic-equilibrium phenomenon associated with the lowering of the GB energy that drives the disordering. Given the rather high GB energies in our model nanocrystalline microstructures, the appearance of an amorphous intergranular phase therefore appears to be an equilibrium effect as well.

Second, the random misorientations between the grains chosen in our model nanocrystalline microstructures effectively avoid the formation of low-angle and "special" boundaries since all GBs have both tilt and twist components and hence a relatively high energy; however, the structures of these (high-energy) boundaries are indistinguishable from the "constrainedamorphous" structures of extended GBs in bicrystals. The main difference between the GBs in coarse-grained and nanocrystalline microstructures therefore seems to lie in their respective distributions of GB energies: Whereas coarse-grained materials exhibit a broad, more equilibrated 
GB-energy distribution, our idealized nanocrystalline microstructures contain only high-energy GBs.

Given the highly idealized microstructures from which these conclusions were drawn, a number of questions remain. For example, one wonders how our results would change upon consideration of more realistic microstructures, including distributions in grain size, grain shapes and grain misorientations. From experiments it would be interesting to know the degree to which the grain misorientations in actual nanocrystalline materials are truly random and the degree to which agglomeration, sintering and grain growth lead to the formation of lowerenergy, and hence crystalline, GBs. Also, in nanocrystalline materials in which an amorphous GB component can be identified, one might ask if or how the fraction of amorphous material correlates with the GB-energy distribution function. While with rapidly increasing computer resources one can hope to address some of these questions by simulation, some key questions can probably not be resolved until better characterized experimental microstructures are available.

ACKNOWLEDGMENTS. PK gratefully acknowledges support from the A. v. Humboldt Foundation. SRP and DW are supported by the US Department of Energy, BES-Materials Science under Contract No. W-31-109-Eng-38.

\section{REFERENCES}

1. T. D. Shen et al., J. Mater. Res. 10, 139 (1995).

2. S. Veprek, Z. Iqbal, H. R. Oswald and A. P. Webb, J. Phys. C 14, 295 (1981).

3. S. R. Phillpot, D. Wolf and H. Gleiter, J. Appl. Phys. 78, 847 (1995).

4. S. R. Phillpot, D. Wolf and H. Gleiter, Scripta Metall. Mater. 33, 1245 (1995).

5. W. Rosenhain and J. C. W. Humfrey, J. of the Iron and Steel Institute 87, 219 (1913); W. Rosenhain and D. Ewen, J. of the Institute of Metals 10, 119 (1913); for an excellent review, see K. T. Aust and B. Chalmers, in Metal Interfaces, (ASM, 1952), p. 153.

6. D. Wolf, J. Wang, S. R. Phillpot, and H. Gleiter, Phys. Rev. Lett. 13, 517 (1996).

7. J. Wang, D. Wolf, S. R. Phillpot, and H. Gleiter, Phil. Mag. 74, 4786 (1995).

8. F. H. Stillinger and T. A. Weber, Phys. Rev. B 31, 5262 (1985).

9. J. Q. Broughton, and X. P. Li, Phys. Rev. B 35, 9120 (1987).

10. M. D. Kluge, J. R. Ray and A. Rahman, Phys. Rev. B 36, 4234 (1987).

11. W. D. Luedtke and U. Landman, Phys. Rev. B 37, 4656 (1988); ibid. 40, 1164 (1989).

12. B. P. Feuston, R. K. Kalia and P. Vashishta, Phys. Rev. B 35, 6222 (1987).

13. S. R. Phillpot and D. Wolf, Phil. Mag. A 60, 545 (1989).

14. V. V. Bulatov, S. Yip and A. S. Argon, Phil. Mag. A 72, 453 (1995).

15. U. Landman et al., Phys. Rev. Lett. 56, 155 (1986).

16. M. D. Kluge and J. R. Ray, Phys. Rev. B 39, 1738 (1989).

17. P. Keblinski, S. R. Phillpot, D. Wolf and H. Gleiter, Acta. Mat. (in press).

18. See, for example, D. Wolf and K. L. Merkle in Materials Interfaces: Atomic-Level Structure and Properties, edited by D. Wolf and S. Yip (Chapman and Hall, 1992), p. $87 \mathrm{ff}$.

19. P. Keblinski, S. R. Phillpot, D. Wolf and H. Gleiter, Acta. Mat. (submitted).

20. M. R. Fitzsimmons et al., Phys. Rev. B 44, 2452 (1991).

21. P. Keblinski, S. R. Phillpot, D. Wolf and H. Gleiter, J. Amer. Ceram. Soc. (submitted).

22. A. Bourret and J. J. Bacmann, Surface Science 162, 495 (1985). 